University of Nebraska - Lincoln

DigitalCommons@University of Nebraska - Lincoln

7-22-2011

\title{
Perturbation-theory analysis of ionization by a chirped few-cycle attosecond pulse
}

\author{
Evgeny A. Pronin \\ University of Nebraska - Lincoln, epronin2@unl.edu \\ Anthony F. Starace \\ University of Nebraska-Lincoln, astarace1@unl.edu \\ Liang-You Peng \\ Peking University
}

Follow this and additional works at: https://digitalcommons.unl.edu/physicsstarace

Part of the Physics Commons

Pronin, Evgeny A.; Starace, Anthony F.; and Peng, Liang-You, "Perturbation-theory analysis of ionization by a chirped few-cycle attosecond pulse" (2011). Anthony F. Starace Publications. 184.

https://digitalcommons.unl.edu/physicsstarace/184

This Article is brought to you for free and open access by the Research Papers in Physics and Astronomy at DigitalCommons@University of Nebraska - Lincoln. It has been accepted for inclusion in Anthony F. Starace Publications by an authorized administrator of DigitalCommons@University of Nebraska - Lincoln. 


\title{
Perturbation-theory analysis of ionization by a chirped few-cycle attosecond pulse
}

\author{
E. A. Pronin and Anthony F. Starace \\ Department of Physics and Astronomy, University of Nebraska, Lincoln, Nebraska 68588-0299, USA \\ Liang-You Peng \\ State Key Laboratory for Mesoscopic Physics and Department of Physics, Peking University, Beijing 100871, China
}

(Received 29 April 2011; published 22 July 2011)

\begin{abstract}
The angular distribution of electrons ionized from an atom by a chirped few-cycle attosecond pulse is analyzed using perturbation theory (PT), keeping terms in the transition amplitude up to second order in the pulse electric field. The dependence of the asymmetry in the ionized electron distributions on both the chirp and the carrier-envelope phase (CEP) of the pulse are explained using a simple analytical formula that approximates the exact PT result. This approximate formula (in which the chirp dependence is explicit) reproduces reasonably well the chirp-dependent oscillations of the electron angular distribution asymmetries found numerically by Peng et al. [Phys. Rev. A 80, 013407 (2009)]. It can also be used to determine the chirp rate of the attosecond pulse from the measured electron angular distribution asymmetry.
\end{abstract}

DOI: 10.1103/PhysRevA.84.013417

PACS number(s): $32.80 . \mathrm{Fb}, 32.80 . \mathrm{Rm}, 32.80 . \mathrm{Qk}$

\section{INTRODUCTION}

Attosecond science is a rapidly developing branch of atomic physics that is providing a deeper understanding of nature by enabling new kinds of probes of electron dynamics in atoms and molecules (see, e.g., Refs. [1-7]). One of its ultimate goals is to use this understanding to control electron motion in atoms and molecules. Few-cycle attosecond pulses are produced by high-order harmonic generation (HHG) in gases exposed to strong infrared (IR) laser radiation of femtosecond duration $[8,9]$, so advances in attosecond science are intimately connected to advances in the generation of intense, short, and stable IR laser pulses [10]. Current methods of generating attosecond pulses introduce a chirp [11,12]. Thus, it is important to investigate the influence of this chirp on the interaction of attosecond pulses with matter in order to both understand its effect on ionized electron angular distributions and then to use this knowledge for better control of electron motion. In the IR frequency domain short, chirped pulses have already been used in a number of applications, such as to control the populations of molecular vibrational and rotational states (see, e.g., Refs. [13-18]), to control ultracold atomic collisions [19], to control population transfer in few-level model atoms [20-23], to control the extent of the plateau cutoffs for both HHG [24,25] and above-threshold ionization (ATI) [26], to control the HHG process in order to produce single attosecond pulses [27], and to increase the intensity of the HHG spectrum using a two-color pump scheme [28]. Both ATI [29] and multiphoton ionization [30,31] by a short, chirped IR pulse have been found to be sensitive to the chirp of the pulse. The process of laser-assisted ionization by a chirped extreme ultraviolet (XUV) pulse has also been analyzed in Refs. [32,33], and the effect of the chirp on the ionization signal was found to be significant. A theoretical analysis of the photoelectron spectrum resulting from ionization of a coherent superposition of two states of the $\mathrm{H}$ atom by a chirped many-cycle attosecond pulse has shown that attosecond time-scale electron dynamics can be measured with even a long attosecond pulse [34]. Recently double ionization of the He atom by a chirped attosecond XUV pulse was investigated by solving numerically the two-electron, time-dependent Schrödinger equation (TDSE); it was found that the single and double electron energy spectra are quite sensitive to the pulse chirp [35].

In this paper we present a time-dependent perturbation theory treatment of the ionization of an atomic system by a single, chirped, few-cycle attosecond XUV pulse that provides a parametrization of the photoelectron angular distribution in terms of the carrier-envelope phase (CEP), the polarization, and the chirp of the pulse. This work generalizes the analysis presented in Ref. [36] by focusing on the effects of the pulse chirp, which were not considered in Ref. [36]. This work aims also to provide an analytical interpretation of the numerical results in Ref. [37] obtained by solving the three-dimensional TDSE for ionization of the $\mathrm{H}$ atom by a few-cycle, chirped attosecond XUV pulse. Our focus is on the chirp-dependent features of those results, especially concerning asymmetries in the photoelectron angular distributions, for the case of a linearly polarized XUV pulse having a Gaussian pulse envelope. In Sec. II we present a brief review of the timedependent perturbation theory approach employed in Ref. [36] and emphasize those new aspects introduced by a nonzero pulse chirp. We note that a perturbation theory treatment of the atom-XUV pulse interaction should be valid up to intensities of order $\sim 10^{16} \mathrm{~W} / \mathrm{cm}^{2}$ [38]. In Sec. III we present a comparison of the results of our theoretical parametrization of the electron angular distribution asymmetry resulting from ionization of atomic $\mathrm{H}$ by a few-cycle, chirped attosecond XUV pulse with the results of the direct solution of the TDSE presented in Ref. [37]. For the case of a linearly polarized, chirped pulse having a Gaussian envelope, we present a simple analytical formula for the chirp dependence of the ionized electron asymmetry. Finally, in Sec. IV we summarize our results and present our conclusions.

\section{THEORETICAL FORMULATION}

We consider the electric-dipole interaction of an atom with a few-cycle, linearly polarized, chirped XUV pulse having a 
form similar to that used by others [29,30,34]. Specifically, the vector potential of the pulse is given by

$$
\mathbf{A}(t)=A_{0}(t) \mathbf{e}_{z} \cos \left[\omega(t) t+\phi_{0}\right]
$$

where $\phi_{0}$ is the CEP of the pulse, $\mathbf{e}_{z}$ is a unit vector along the laser field polarization axis, and $A_{0}(t)$ is the envelope of the pulse, which we assume to have the following Gaussian shape:

$$
A_{0}(t)=\frac{c \sqrt{I_{0}}}{\omega_{0}\left(1+\xi^{2}\right)^{1 / 4}} \exp \left(-4 \ln 2 \frac{t^{2}}{\tau_{0}^{2}}\right) .
$$

In Eq. (1) the frequency $\omega(t)$ is assumed to be linearly chirped, i.e.,

$$
\omega(t)=\omega_{0}+4 \ln 2 \frac{\xi}{1+\xi^{2}} \frac{t}{\tau_{0}^{2}},
$$

where $\xi$ is the chirp rate. The remaining parameters in Eqs. (1), (2), and (3) are most easily defined for the case of $\xi=0$, which corresponds to a transform-limited pulse having peak intensity $I_{0}$, carrier frequency $\omega_{0}$, and duration $\tau_{0}$ (full width at half maximum). A chirped pulse has a larger duration, $\tau=$ $\tau_{0} \sqrt{1+\xi^{2}}$, and a smaller peak intensity, $I=I_{0} / \sqrt{1+\xi^{2}}$, but the total energy per pulse remains the same as for a transformlimited pulse.

In our analysis it is useful to define the Fourier transform of the pulse electric field,

$$
\hat{\mathbf{F}}(\varepsilon)=\int_{-\infty}^{\infty} \mathbf{F}(t) e^{i \varepsilon t} d t
$$

where the electric field is given by $\mathbf{F}(t)=-\frac{1}{c} \frac{\partial \mathbf{A}(t)}{\partial t}$. The Fourier transforms of $\mathbf{F}$ and $\mathbf{A}$ [where $\lim _{t \rightarrow \pm \infty} \mathbf{A}(t) \stackrel{c}{=} 0$ ] satisfy the following relation:

$$
\hat{\mathbf{F}}(\varepsilon)=\frac{i \varepsilon}{c} \hat{\mathbf{A}}(\varepsilon) .
$$

Moreover, $\hat{\mathbf{F}}(\varepsilon)$ can be written as a sum of two complex terms,

$$
\hat{\mathbf{F}}(\varepsilon)=\mathbf{e}_{z}\left[e^{-i \phi_{0}} \hat{F}^{+}(\varepsilon)+e^{i \phi_{0}} \hat{F}^{-}(\varepsilon)\right],
$$

where the factors $\hat{F}^{ \pm}(\varepsilon)$ are the CEP- and polarizationindependent, scalar parameters of the pulse:

$$
\hat{F}^{ \pm}(\varepsilon)=\frac{i \varepsilon}{2 c} \int A_{0}(t) e^{i[\varepsilon \mp \omega(t)] t} d t, \quad \hat{F}^{-}(\varepsilon)=\left[\hat{F}^{+}(-\varepsilon)\right]^{*} .
$$

For a Gaussian-shaped pulse envelope the Fourier transform of the pulse can be calculated analytically:

$$
\hat{F}^{ \pm}(\varepsilon)=\frac{i \varepsilon}{c} B^{ \pm} e^{-\frac{\left(\omega_{0} \mp \varepsilon\right)^{2}}{4 A^{ \pm}}},
$$

where

$$
A^{ \pm}=\frac{4 \ln 2}{(1 \mp i \xi) \tau_{0}^{2}}, \quad B^{ \pm}=\frac{A_{0}}{2\left(1+\xi^{2}\right)^{1 / 4}} \frac{\sqrt{\pi}}{\sqrt{A^{ \pm}}} .
$$

For $\xi=0$, Eq. (8) takes the form

$$
\hat{F}^{ \pm}(\varepsilon)=\frac{i \varepsilon}{4 c} A_{0} \tau_{0} \sqrt{\frac{\pi}{\ln 2}} e^{-\frac{\tau_{0}^{2}\left(\omega_{0} \mp \varepsilon\right)^{2}}{16 \ln 2}} .
$$

Within the time-dependent PT approach, the probability for electron ionization in the direction $\hat{\mathbf{p}}$ with energy $E=p^{2} / 2$ is given in atomic units (which are employed throughout this paper, unless otherwise noted) by

$$
\begin{aligned}
\mathcal{W} & =\frac{p}{2 l+1} \sum_{m}\left|A_{1}+A_{2}+\cdots\right|^{2} \\
& \approx \frac{p}{2 l+1} \sum_{m}\left[\left|A_{1}\right|^{2}+2 \operatorname{Re}\left(A_{1}^{*} A_{2}\right)+\cdots\right],
\end{aligned}
$$

where $A_{1}$ and $A_{2}$ are the first- and second-order transition amplitudes (in the electric-dipole approximation):

$$
\begin{aligned}
A_{1}= & \left\langle\psi_{\mathbf{p}}^{(-)}\left|\hat{\mathbf{F}}\left(E-E_{n}\right) \cdot \mathbf{r}\right| \psi_{n l m}\right\rangle, \\
A_{2}= & -\frac{1}{2 \pi} \int_{-\infty}^{\infty} d \varepsilon\left\langle\psi_{\mathbf{p}}^{(-)}\right| \hat{\mathbf{F}}(E-\varepsilon) \cdot \mathbf{r} \\
& \times G_{\varepsilon}\left(\mathbf{r}, \mathbf{r}^{\prime}\right) \hat{\mathbf{F}}\left(\varepsilon-E_{n}\right) \cdot \mathbf{r}^{\prime}\left|\psi_{n l m}\right\rangle,
\end{aligned}
$$

where $G_{\varepsilon}$ is a stationary Green function for the electron:

$G_{\varepsilon}\left(\mathbf{r}, \mathbf{r}^{\prime}\right)=\sum_{k l^{\prime} m^{\prime}} \frac{\left|\psi_{k l^{\prime} m^{\prime}}\right\rangle\left\langle\psi_{k l^{\prime} m^{\prime}}\right|}{E_{k}-\varepsilon}+\int d \mathbf{p} \frac{\left|\psi_{\mathbf{p}}^{(-)}\right\rangle\left\langle\psi_{\mathbf{p}}^{(-)}\right|}{p^{2} / 2-\varepsilon-i 0}$.

The amplitudes $A_{1}$ and $A_{2}$ have been expressed using the single active electron approximation, where $\psi_{n l m}$ describes the initial bound state of the electron with energy $E_{n}$ and $\psi_{\mathbf{p}}^{(-)}$ describes the final continuum state (satisfying the incoming wave boundary condition) with electron momentum $\mathbf{p}$ and energy $E=p^{2} / 2$. Both wave functions are solutions of the time-independent Schrödinger equation for an electron in a potential $U(r)$ :

$$
\begin{aligned}
& {\left[-\nabla^{2} / 2+U(r)\right] \psi_{n l m}(\mathbf{r})=E_{n} \psi_{n l m}(\mathbf{r}),} \\
& {\left[-\nabla^{2} / 2+U(r)\right] \psi_{\mathbf{p}}^{(-)}(\mathbf{r})=\frac{p^{2}}{2} \psi_{\mathbf{p}}^{(-)}(\mathbf{r}) .}
\end{aligned}
$$

In Ref. [36] the functional dependence of the ionization probability $\mathcal{W}$ on the CEP, the pulse polarization, and the ejected electron direction $\hat{\mathbf{p}}$ was parametrized ab initio using very general rotational invariance and symmetry arguments. The parametrization results of Ref. [36] apply even when the carrier frequency $\omega$ is time dependent, as in the case of nonzero chirp. In this case only the Fourier transform of the pulse changes, but all other parametrization results for $\mathcal{W}$ remain valid. For a randomly oriented target atom (i.e., unpolarized and unaligned) and for the case that neither the spin state of the electron nor the angular momentum state of the residual ion are measured, the ionization probability (11) for an initial $s$ state by a linearly polarized pulse has the following form (see Ref. [36] for a detailed derivation):

$$
\begin{aligned}
\mathcal{W}= & \mathcal{C}\left[1+\beta_{0}\left(3 \cos ^{2} \alpha-1\right) / 2\right] \\
& +\cos \alpha \operatorname{Re}\left[\left(\gamma_{1}+\gamma_{2} \cos ^{2} \alpha+\gamma_{3}\right) e^{-i \phi_{0}}\right],
\end{aligned}
$$

where $\alpha$ is the angle between the polarization axis and the ionized electron momentum direction, $\hat{\mathbf{p}} ; \mathcal{C}, \beta_{0}$ are two real parameters, and $\gamma_{i}, 1 \leqslant i \leqslant 3$, are three complex scalar parameters. Note that in Eq. (17) the dependence of $\mathcal{W}$ on the CEP, $\phi_{0}$, and the ejected electron direction, $\hat{\mathbf{p}}$, has been made explicit; i.e., the five parameters in this equation are independent of them. The parameters $\mathcal{C}, \beta_{0}$ originate from the first-order PT term $\left|A_{1}\right|^{2}$. They have the following form in 
terms of the Fourier transforms of the pulse and the first-order radial matrix elements:

$$
\begin{aligned}
\mathcal{C} & =\frac{p}{3}\left|\alpha_{+}\right|^{2}, \quad \beta_{0}=2, \\
\alpha_{+} & =\mathcal{A}_{p 1} \hat{F}^{+}\left(E-E_{n}\right), \\
\mathcal{A}_{p 1} & =-\frac{i e^{i \delta_{1}}}{\sqrt{4 \pi} p}\langle p 1|r| n 0\rangle .
\end{aligned}
$$

The parameters $\gamma_{i}$ originate from the interference of the first- and second-order transition amplitudes, $2 \operatorname{Re}\left(A_{1}^{*} A_{2}\right)$. The asymmetry in the ionized electron angular distribution stems only from this interference term in Eq. (17), which is odd in $\cos \alpha$. The parameters $\gamma_{i}$ are thus products of the first- and second-order radial matrix elements:

$$
\begin{gathered}
\gamma_{1}=2 p \alpha_{+} M_{0+2}^{+-*}, \\
\gamma_{2}=-6 p\left(\alpha_{+} M_{2}^{+-*}+\alpha_{+}^{*} M_{2}^{++}\right), \\
\gamma_{3}=2 p \alpha_{+}^{*} M_{0+2}^{++},
\end{gathered}
$$

where

$$
\begin{aligned}
M_{l}^{++}=\int d \varepsilon \hat{F}^{+}(E-\varepsilon) \hat{F}^{+}\left(\varepsilon-E_{n}\right) \mathcal{M}_{p l}(\varepsilon), \\
M_{l}^{+-}=\int d \varepsilon\left[\hat{F}^{+}(E-\varepsilon) \hat{F}^{-}\left(\varepsilon-E_{n}\right)\right. \\
\left.\quad+\hat{F}^{-}(E-\varepsilon) \hat{F}^{+}\left(\varepsilon-E_{n}\right)\right] \mathcal{M}_{p l}(\varepsilon), \\
M_{0+2}^{++}=M_{0}^{++}+M_{2}^{++}, \quad M_{0+2}^{+-}=M_{0}^{+-}+M_{2}^{+-},
\end{aligned}
$$

with

$$
\mathcal{M}_{p l}(\varepsilon)=-\frac{e^{i \delta_{l}}}{12 \pi^{3 / 2} p}\left\langle p l\left|r g_{1}\left(\varepsilon ; r, r^{\prime}\right) r^{\prime}\right| n 0\right\rangle .
$$

In Eq. (27), $g_{1}(\varepsilon)$ is the radial part of the Green function for the atom:

$$
G_{E}\left(\mathbf{r}, \mathbf{r}^{\prime}\right)=\sum_{l m} g_{l}\left(E ; r, r^{\prime}\right) Y_{l m}(\hat{\mathbf{r}}) Y_{l m}^{*}\left(\hat{\mathbf{r}}^{\prime}\right) .
$$

For the case of ionization of the $1 s$ state of the $\mathrm{H}$ atom to a continuum state with energy $E=p^{2} / 2$, the first- and second-order radial matrix elements (20) and (27) have known analytic forms (see Appendix B in Ref. [36]). The asymmetry in the ionized electron angular distribution is maximal along the polarization axis and can be written as follows:

$$
\begin{gathered}
\left.\Delta \mathcal{W} \equiv \mathcal{W}\right|_{\alpha=0}-\left.\mathcal{W}\right|_{\alpha=\pi}=2 \operatorname{Re}\left[\left(\gamma_{1}+\gamma_{2}+\gamma_{3}\right) e^{-i \phi_{0}}\right] \\
=2\left|\gamma_{1}+\gamma_{2}+\gamma_{3}\right| \cos \left(\phi_{0}-\Theta_{L}\right)
\end{gathered}
$$

where $\Theta_{L} \equiv \arg \left[\gamma_{1}+\gamma_{2}+\gamma_{3}\right]$.

\section{RESULTS AND DISCUSSION}

In Fig. 1 we present our PT results for the ionized electron asymmetry $\Delta \mathcal{W}(29)$ resulting from ionization of the $1 s$ state of the $\mathrm{H}$ atom by a single-cycle XUV pulse with $\omega=25 \mathrm{eV}$, peak intensity $I_{0}=10^{14} \mathrm{~W} / \mathrm{cm}^{2}$, and four different chirp rates: $\xi=$ $0,0.75,1.5$, and 3.5. These PT results are compared with the results of Ref. [37], which are obtained by numerically solving the three-dimensional, time-dependent Schrödinger equation. One sees that the agreement is quite good, validating our use
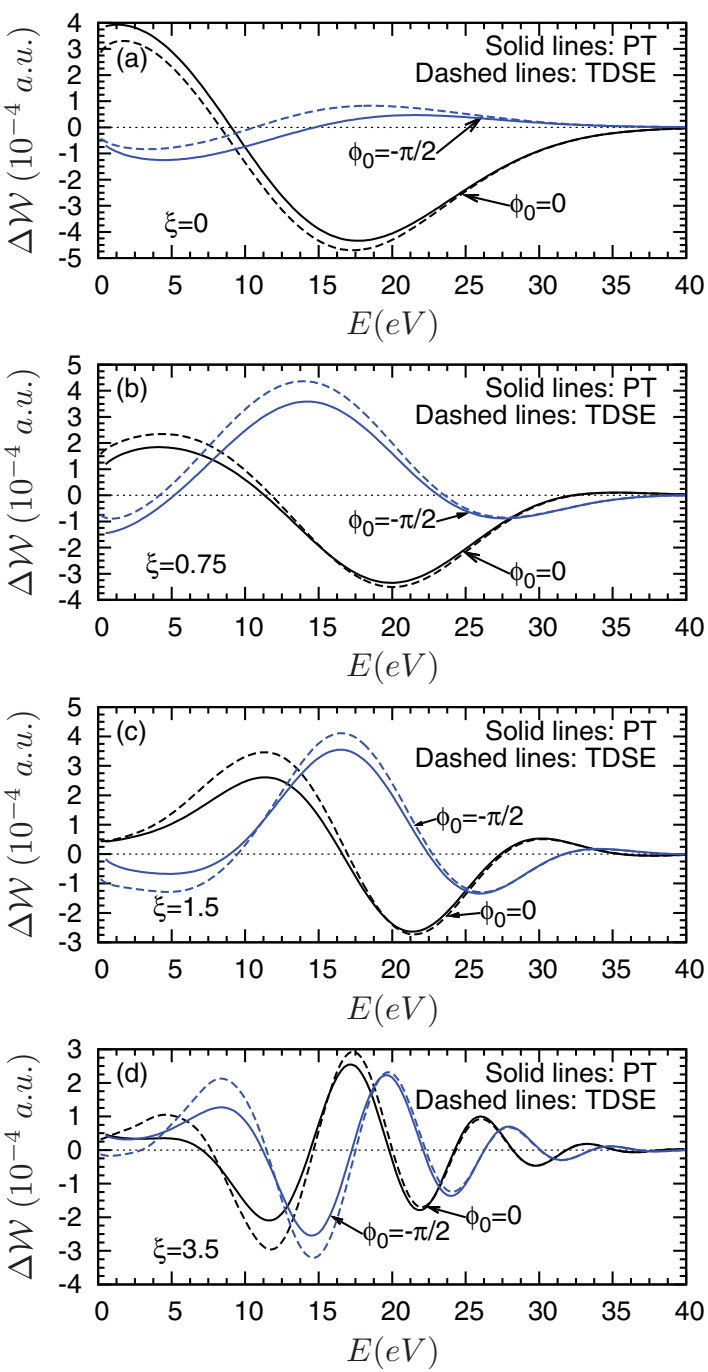

FIG. 1. (Color online) Comparison of the present PT results with TDSE results of Ref. [37] for the ionized electron asymmetry $\Delta \mathcal{W}$ [see Eqs. (29) and (30)] for electrons ionized from the $\mathrm{H}$ atom along the laser polarization axis by a linearly polarized, single-cycle XUV Gaussian pulse with carrier frequency $\omega=25$ $\mathrm{eV}$, CEPs $\phi_{0}=0$ and $\phi_{0}=-\pi / 2$, peak intensity $I_{0}=10^{14} \mathrm{~W} / \mathrm{cm}^{2}$, one cycle duration $\tau_{0}=T_{0}$, and four different chirp rates: $\xi=$ (a) 0 ,(b) 0.75 , (c) 1.5 ,(d) 3.5 .

of PT. The differences in the results can be attributed in part to higher-order PT terms that are neglected in our perturbative analysis, such as, e.g., the interference of the second- and thirdorder PT amplitudes, and in part to numerical inaccuracies in both the PT and TDSE calculations. Quantitatively, we estimate that the contributions of the higher-order PT terms at energies of about 0.5 a.u. $\approx 13.6 \mathrm{eV}$ contribute about $1 \%$ to the ionization probability and that numerical errors in calculating the second-order PT amplitude or in solving the TDSE are also around $1 \%$. Thus agreement between the PT results and the TDSE results is excellent for the ionization probabilities along the axis of laser polarization. (This excellent agreement has been shown for the case of transform-limited pulses in Fig. 2 of Ref. [36]; comparisons for pulses having a nonzero chirp show similarly excellent agreement.) In calculating the asymmetry, however, the differences are increased owing to the subtraction 
of probabilities of comparable magnitudes, resulting in the somewhat larger differences at particular energies shown in Fig. 1. Nevertheless, the quantitative agreement is quite good.

The results in Fig. 1 display several notable features: (1) For $\xi=0$, the magnitude of the asymmetry $\Delta \mathcal{W}$ for CEP $\phi_{0}=-\pi / 2$ is significantly smaller than for $\phi_{0}=0$. (2) As the chirp rate increases, the number of sign changes of the asymmetry $\Delta \mathcal{W}$ increases. (3) The asymmetry $\Delta \mathcal{W}$ for CEP $\phi_{0}=0$ is shifted approximately by a phase $\pi / 2$ with respect to that for a CEP $\phi_{0}=-\pi / 2$ (i.e., the zeros of one occur approximately at the maxima or minima of the other, and vice versa; this fact is especially pronounced for higher chirp rates). To explain these features we must analyze in more detail the dependence of the PT expression for the asymmetry $\Delta \mathcal{W}(29)$ on the CEP, $\phi_{0}$, and the chirp rate, $\xi$.

The dependence of the asymmetry $\Delta \mathcal{W}$ on the chirp rate is implicit in the PT expressions (29) and (30), stemming from the parameters $\gamma_{i}, 1 \leqslant i \leqslant 3$, defined in Eqs. (21)-(23). These parameters in turn depend on the integrals defined in Eqs. (24) and (25), whose integrands involve the Fourier transforms of the pulse with second-order radial matrix elements of the electric dipole operator. Owing to the presence of the latter matrix elements, it is not possible in general to evaluate the integral analytically (and thus to extract the explicit dependence of $\Delta \mathcal{W}$ on the chirp rate). If, however, one assumes that the integrands in Eqs. (24) and (25) depend on energy primarily through the exponential factor in the Fourier transforms $\hat{F}^{ \pm}$(cf. Eq. (8)) (i.e., if we assume that $-\frac{(E-\epsilon)\left(\epsilon-E_{n}\right)}{c^{2}} \mathcal{M}_{p l} \approx$ const $\left.\equiv \overline{\mathcal{M}}_{l}\right)$, then an analytic integration becomes possible. Using expression (8) for the Fourier transform of the pulse, the analytic results for the integrals in Eqs. (24) and (25) are

$$
\begin{gathered}
M_{l}^{++}=\overline{\mathcal{M}}_{l}^{++} \sqrt{2 \pi A^{+}}\left(B^{+}\right)^{2} e^{-\frac{\left(E_{i}-E_{f}+2 \omega_{0}\right)^{2}}{8 A^{+}}}, \\
M_{l}^{+-}=2 \overline{\mathcal{M}}_{l}^{+-} \sqrt{\pi} B^{+} B^{-} \sqrt{\frac{A^{+} A^{-}}{A^{+}+A^{-}}} e^{-\frac{\left(E_{f}-E_{i}\right)^{2}}{4\left(A^{+}+A^{-}\right)}},
\end{gathered}
$$

where we have labeled the initial and final state energies explicitly as $E_{i} \equiv E_{n}$ and $E_{f} \equiv E=p^{2} / 2$. For both consistency and simplicity, we assume similarly that the first-order amplitude, $\alpha_{+}$, in Eq. (19) depends on energy primarily through the exponential factor in the Fourier transform $\hat{F}^{+}\left(E_{f}-E_{i}\right)$ [see Eq. (8)] [i.e., that $(i \varepsilon / c) \mathcal{A}_{p 1} \approx$ const $\equiv \overline{\mathcal{A}}_{1}$ ] so that Eq. (19) becomes

$$
\alpha_{+}=\overline{\mathcal{A}}_{1} B^{+} e^{-\frac{\left(E_{i}-E_{f}+\omega_{0}\right)^{2}}{4 A^{+}}}
$$

With these assumptions, the asymmetry $\Delta \mathcal{W}$ in Eq. (29) takes the following approximate form, whose virtue is that the dependence on the chirp rate, $\xi$, is explicit:

$$
\begin{aligned}
\Delta \mathcal{W}= & \frac{p A_{0}^{3} \tau_{0}^{2}}{\left(1+\xi^{2}\right)^{1 / 4}} e^{-\Delta_{1}^{2}} \operatorname{Re}\left(e ^ { - i \phi _ { 0 } } \left\{C_{1} \sqrt{1-i \xi}\right.\right. \\
& \times \exp \left[i \xi \Delta_{1}^{2}-\left(1+\xi^{2}\right) \Delta_{0}^{2} / 2\right] \\
& \left.\left.+C_{2} \exp 0\left[-i \xi \Delta_{1}^{2}-(1-i \xi) \Delta_{2}^{2} / 2\right]\right\}\right),
\end{aligned}
$$

where

$$
\Delta_{n} \equiv\left(E_{i}-E_{f}+n \omega_{0}\right) \frac{\tau_{0}}{4 \sqrt{\ln 2}},
$$

$n$ is the number of photons absorbed, and $C_{1}$ and $C_{2}$ are two complex constants, whose expressions in terms of the approximate second- and first-order amplitudes [see Eqs. (31), (32), and (33)] are as follows:

$$
\begin{aligned}
& C_{1}=\overline{\mathcal{A}}_{1}\left(\overline{\mathcal{M}}_{0}^{+-}-2 \overline{\mathcal{M}}_{2}^{+-}\right)^{*} \frac{\pi^{2}}{4 \sqrt{2} \ln 2}, \\
& C_{2}=\overline{\mathcal{A}}_{1}^{*}\left(\overline{\mathcal{M}}_{0}^{++}-2 \overline{\mathcal{M}}_{2}^{++}\right) \frac{\pi^{2}}{4 \sqrt{2} \ln 2} .
\end{aligned}
$$

In practice, these two constants can be determined best by matching the approximate formula (34) either to results of a numerical evaluation of the exact PT result in Eq. (29) or to TDSE results; alternatively, they can be determined by matching to results of an experimental measurement. Carrying out such a matching at four different energies will give four linear equations that determine the four unknown parameters, i.e., the real and imaginary parts of $C_{1}$ and $C_{2}$.

Expression (34) is simpler to analyze if we explicitly take the real part of it to obtain

$$
\begin{aligned}
\Delta \mathcal{W}= & \frac{p A_{0}^{3} \tau_{0}^{2}}{\left(1+\xi^{2}\right)^{1 / 4}} e^{-\Delta_{1}^{2}}\left\{\left|C_{1}\right|\left(1+\xi^{2}\right)^{1 / 4} e^{-\left(1+\xi^{2}\right) \Delta_{0}^{2} / 2}\right. \\
& \times \cos \left[-\phi_{0}+\arg \left(C_{1}\right)+\arg (1-i \xi) / 2+\xi \Delta_{1}^{2}\right] \\
& \left.+\left|C_{2}\right| e^{-\Delta_{2}^{2} / 2} \cos \left[\phi_{0}-\arg \left(C_{2}\right)+\xi \Delta_{1}^{2}-\xi \Delta_{2}^{2} / 2\right]\right\} .
\end{aligned}
$$

The overall factor $e^{-\Delta_{1}^{2}}$ provides a Gaussian envelope for the asymmetry $\Delta \mathcal{W}$ that is centered at the energy for onephoton absorption, $E_{f}=E_{i}+\omega_{0}$. The term corresponding to absorption plus emission and emission plus absorption is centered about $E_{f}=E_{i}$ (see the term $\sim e^{-\left(1+\xi^{2}\right) \Delta_{0}^{2} / 2}$ ); it thus contributes mostly to the low-energy part of the electron energy distribution. The term corresponding to two-photon absorption is centered at $E_{f}=E_{i}+2 \omega_{0}$ (cf. the term $\sim e^{-\Delta_{2}^{2} / 2}$ ); it contributes mostly to the high-energy part of the electron energy distribution. The terms in the arguments of the two cosine functions that are proportional to $\sim \xi \Delta_{n}^{2}$ obviously do not contribute in the case of zero chirp; for nonzero chirp, however, they are responsible for an increased oscillation frequency of the asymmetry $\Delta \mathcal{W}$ with energy as the chirp rate $\xi$ increases, in agreement with the results shown in Fig. 1. Finally, Eq. (37) makes obvious the $\pi / 2$ phase difference shown in Fig. 1 between the asymmetries $\Delta \mathcal{W}$ for CEPs of $\phi_{0}=0$ and $\phi_{0}=-\pi / 2$.

Further analysis of the chirp-dependent features of the asymmetry $\Delta \mathcal{W}$ shown in Fig. 1 requires knowing the values of the complex constants $C_{1}$ and $C_{2}$ for the laser parameters used in that figure. We determined these constants by matching the results of our numerical evaluation of the exact PT result in Eq. (29) to the approximate formula (37) at two different energies for each of two different CEPs (with $\xi=0$ in all four cases): (1) $E_{f}=2.5 \mathrm{eV}, \phi_{0}=-\pi / 2$, (2) $E_{f}=19.5 \mathrm{eV}, \phi_{0}=-\pi / 2$, (3) $E_{f}=3.5 \mathrm{eV}, \phi_{0}=0$, and (4) $E_{f}=18.5 \mathrm{eV}, \phi_{0}=0$. The choices of the matching points are quite obvious: We need low-energy points to determine with sufficient accuracy the constant $C_{1}$ for the emissionabsorption term; correspondingly, higher-energy points are needed to describe the region where two-photon absorption contributes significantly so as to determine $C_{2}$ accurately. In addition, carrying out the fitting using two CEPs, $\phi_{0}=0$ 

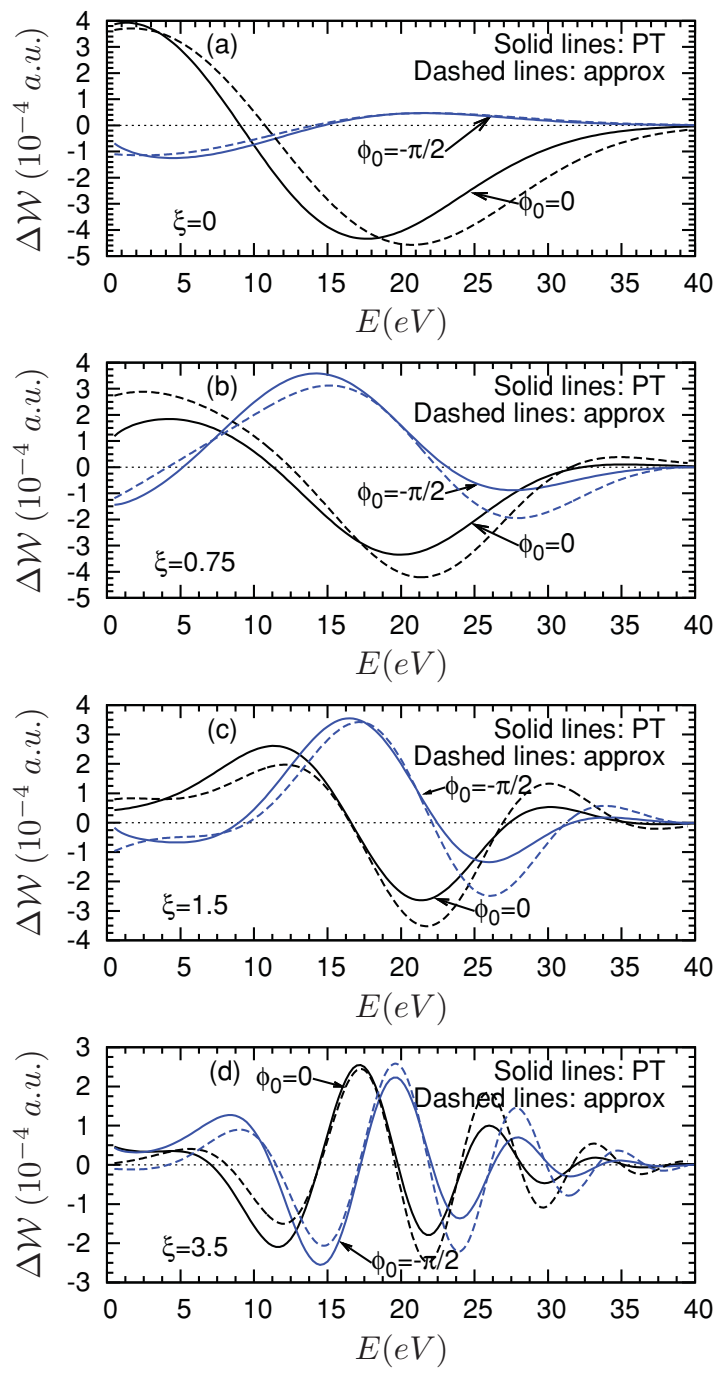

FIG. 2. (Color online) Comparison of results for the asymmetry $\Delta \mathcal{W}$ obtained using the exact PT formula (29) with those obtained using the approximate formula (37). The parameters of the laser pulse are the same as for the results in Fig. 1.

and $\phi_{0}=-\pi / 2$, ensures that the matching procedure gives appropriate values of the constants. By this fitting procedure, we obtained $C_{1}=(9-2.63 i) \times 10^{-9}=9.38 \times 10^{-9} e^{-0.09 i \pi}$ and $C_{2}=(-10.81-1.21 i) \times 10^{-9}=10.88 \times 10^{-9} e^{-0.96 i \pi}$. We note that since $\arg \left(C_{1}\right)$ is relatively small and $\arg \left(C_{2}\right) \approx$ $-\pi$, for a CEP $\phi_{0}=-\pi / 2$ and $\xi=0$ both $\cos \left[-\phi_{0}+\right.$ $\left.\arg \left(C_{1}\right)\right]$ and $\cos \left[\phi_{0}-\arg \left(C_{2}\right)\right]$ are $\approx \cos (\pi / 2)$, so that the asymmetry $\Delta \mathcal{W}$ is small for any final state energy, as is in fact seen in Fig. 1(a). However, if the chirp is nonzero, the asymmetry for a CEP $\phi_{0}=-\pi / 2$ is not small anymore due to the presence of additional chirp-dependent phases.

With the values of the constants $C_{1}$ and $C_{2}$ given above (and determined for the case $\xi=0$ ), the approximate formula (37) gives results for all chirp rates that are in good agreement (both quantitatively and qualitatively) with the results of a numerical evaluation of the exact PT expression (29), as shown in Fig. 2. We note also that in order to describe the asymmetry $\Delta \mathcal{W}$ for higher final state electron energies $\left(E_{i}+\omega_{0}<E_{f}<E_{i}+\right.$ $2 \omega_{0}$, i.e., $11.4 \mathrm{eV}<E_{f}<36.4 \mathrm{eV}$ for the laser parameters used in Figs. 1 and 2), only the two-photon absorption term in Eq. (37) is sufficient [i.e. we can put $C_{1}=0$ in (37)]. Thus, the asymmetry would have the following form:

$$
\begin{aligned}
\Delta \mathcal{W}_{E_{i}+\omega_{0}<E_{f}<E_{i}+2 \omega_{0}} & \frac{p A_{0}^{3} \tau_{0}^{2}}{\left(1+\xi^{2}\right)^{1 / 4}}\left|C_{2}\right| e^{-\Delta_{1}^{2}-\Delta_{2}^{2} / 2} \\
& \times \cos \left[\phi_{0}-\arg \left[C_{2}\right]+\xi\left(\Delta_{1}^{2}-\Delta_{2}^{2} / 2\right)\right] .
\end{aligned}
$$

In this case, matching the period of the experimentally measured asymmetry oscillations with that of the cosine function in (38) provides a means to determine the chirp rate of the pulse.

\section{SUMMARY AND CONCLUSIONS}

In conclusion, we have presented an exact PT formula (29) for the asymmetry of electrons ionized parallel and antiparallel to the direction of linear polarization of a chirped, few-cycle attosecond pulse. For the case of chirped, few-cycle XUV ionization of the $\mathrm{H}$ atom, the results obtained from the exact PT formula are in good agreement with results of the TDSE calculations of Ref. [37]. In addition, we have derived an approximate analytic formula (37) in which the chirp dependence is explicit. This simple analytical formula (37) has allowed us to explain all of the main features of the asymmetry $\Delta \mathcal{W}$ shown in Fig. 1. Moreover, by fitting two complex parameters in that approximate formula, we are able to reproduce well the results obtained numerically by evaluating the exact PT formula in Eq. (29).

\section{ACKNOWLEDGMENTS}

This work was supported in part by the US Department of Energy, Office of Science, Division of Chemical Sciences, Geosciences, and Biosciences, under Grant No. DE-FG0396ER14646, and by the National Natural Science Foundation of China under Grant Nos. 10974007 and 10821062.
[1] P. B. Corkum and F. Krausz, Nature Phys. 3, 381 (2007).

[2] F. Krausz and M. Ivanov, Rev. Mod. Phys. 81, 163 (2009).

[3] G. L. Yudin, S. Chelkowski, J. Itatani, A. D. Bandrauk, and P. B. Corkum, Phys. Rev. A 72, 051401(R) (2005).

[4] S. Chelkowski, G. L. Yudin, and A. D. Bandrauk, J. Phys. B 39, S409 (2006).

[5] T. Morishita, S. Watanabe, and C. D. Lin, Phys. Rev. Lett. 98, 083003 (2007).
[6] M. Uiberacker et al., Nature (London) 446, 627 (2007).

[7] J. Itatani, J. Levesque, D. Zeidler, H. Niikura, H. Pépin, J. C. Kieffer, P. B. Corkum, and D. M. Villeneuve, Nature (London) 432, 867 (2004).

[8] G. Sansone et al., Science 314, 443 (2006).

[9] E. Goulielmakis et al., Science 320, 1614 (2008).

[10] T. Brabec and F. Krausz, Rev. Mod. Phys. 72, 545 (2000).

[11] S. Kazamias and P. Balcou, Phys. Rev. A 69, 063416 (2004). 
[12] Z. Chang, Phys. Rev. A 71, 023813 (2005).

[13] S. Chelkowski and A. D. Bandrauk, J. Raman Spectrosc. 28, 459 (1997).

[14] T. Hornung, R. Meier, and M. Motzkus, Chem. Phys. Lett. 326, 445 (2000).

[15] F. Légaré, S. Chelkowski, and A. D. Bandrauk, Chem. Phys. Lett. 329, 469 (2000).

[16] V. S. Malinovsky and J. L. Krause, Eur. Phys. J. D 14, 147 (2001).

[17] C. Sarkar, R. Bhattacharya, S. S. Bhattacharyya, and S. Saha, Phys. Rev. A 78, 023406 (2008).

[18] J. Plenge, A. Wirsing, C. Raschpichler, M. Meyer, and E. Rühl, J. Chem. Phys. 130, 244313 (2009).

[19] M. J. Wright, J. A. Pechkis, J. L. Carini, S. Kallush, R. Kosloff, and P. L. Gould, Phys. Rev. A 75, 051401 (2007).

[20] R. Marani and E. J. Robinson, J. Phys. B 32, 711 (1999).

[21] B. Chatel, J. Degert, S. Stock, and B. Girard, Phys. Rev. A 68, 041402(R) (2003).

[22] G. P. Djotyan, J. S. Bakos, Z. Sörlei, and J. Szigeti, Phys. Rev. A 70, 063406 (2004).

[23] S. A. Malinovskaya, Phys. Rev. A 73, 033416 (2006).

[24] Y. Xiang, Y. Niu, and S. Gong, Phys. Rev. A 79, 053419 (2009).

[25] J. Xu, B. Zeng, and Y. Yu, Phys. Rev. A 82, 053822 (2010).
[26] Y. Xiang, Y. Niu, and S. Gong, Phys. Rev. A 80, 023423 (2009).

[27] Y. Niu, Y. Xiang, Y. Qi, and S. Gong, Phys. Rev. A 80, 063818 (2009).

[28] R. A. Ganeev, H. Singhal, P. A. Naik, I. A. Kulagin, P. V. Redkin, J. A. Chakera, M. Tayyab, R. A. Khan, and P. D. Gupta, Phys. Rev. A 80, 033845 (2009).

[29] T. Nakajima, Phys. Rev. A 75, 053409 (2007).

[30] T. Nakajima and E. Cormier, Opt. Lett. 32, 2879 (2007).

[31] M. J. Abel, T. Pfeifer, A. Jullien, P. M. Nagel, M. J. Bell, D. M. Neumark, and S. R. Leone, J. Phys. B 42, 075601 (2009).

[32] G. L. Yudin, S. Patchkovskii, and A. D. Bandrauk, J. Phys. B 41, 045602 (2008).

[33] E. E. Krasovskii and M. Bonitz, Phys. Rev. A 80, 053421 (2009).

[34] G. L. Yudin, A. D. Bandrauk, and P. B. Corkum, Phys. Rev. Lett. 96, 063002 (2006).

[35] T.-G. Lee, M. S. Pindzola, and F. Robicheaux, Phys. Rev. A 79, 053420 (2009).

[36] E. A. Pronin, A. F. Starace, M. V. Frolov, and N. L. Manakov, Phys. Rev. A 80, 063403 (2009).

[37] L. Y. Peng, F. Tan, Q. Gong, E. A. Pronin, and A. F. Starace, Phys. Rev. A 80, 013407 (2009).

[38] L. A. A. Nikolopoulos and P. Lambropoulos, Phys. Rev. A 74, 063410 (2006). 